9th International Conference on Industrial Engineering and Industrial Management XXI International Conference on Industrial Engineering and Operations Management International IIE Conference 2015

Aveiro, Portugal. July 6-8, 2015

\title{
Productivity improvement, considering legal conditions and Just In Time principles in the mixed-model Sequencing problem
}

\author{
Bautista $\mathbf{J}^{1}$, Alfaro-Pozo $\mathbf{R}^{\mathbf{1}}$, Batalla-García $\mathbf{C}^{\mathbf{1}}$
}

\begin{abstract}
A new mathematical model to solve the Mixed-Model Sequencing Problem with Work overload minimization is formulated. The model incorporates productive, social and legal aspects in order to move the theory problem closer to the actual industrial environments. Specifically, there are considered the variation of work pace of workers throughout the workday to increase the completed work; the conditions of occupancy level of workers imposed by the collective agreements; and the idea of keeping constant the production mix through the sequence leading both to a balance between the required workloads at stations and regular consumption of components. Indeed, by means of a case study linked to Nissan, a gain of over $98 \%$ is achieved in terms of regular cumulative production and required work, while performing the $100 \%$ of required work and following legal restrictions of operators' saturation.
\end{abstract}

Keywords:Mixed-model; sequencing; just-in-time; work pace; saturation

\section{Introduction}

The flexible assembly systems, such as the Mixed-Model Assembly Lines (MMALs), are characterized by the manufacturing of a set $I$ of products types. Althought products are from the same family, they may require different material consumption and resource use, such as different processing times of operations.

\footnotetext{
${ }^{1}$ Joaquín Bautista Valhondo; Rocío Alfaro Pozo; Cristina Batalla García ( $\square$ email:\{joaquin.bautista; rocio.alfaro; cristina.batalla\}@upc.edu) Research Group OPEPROTHIUS. Dpto. de Organización de Empresas. Universitat Politècnica de Catalunya. Avda. Diagonal, 647, 7th floor, 08028 Barcelona, Spain.

This work is supported by the Ministerios Españoles de Educación y Ciencia and Economía y Competitividad under Projects DPI2010-16759 and TIN2014-57497 including EDRF fundings.
} 
In the MMALs the product units are moved from one workstation to the next by means of a conveyor system with a constant speed. Furthermore, all products keep the same distance between them so that each workstation has the same time (at normal activity) to perform its workload. This time, named cycle time, $c$, may be smaller o larger than the processing time of operations depending of the product type and therefore the station may be under or overloaded. For this reason, it is necessary to sequence the units of the demand plan with the aim of minimizing the unproductive time or the work overload of the line.

In this work, the Mixed-Model Sequencing Problem is studied, considering the general optimization criteria proposed by Boysen (2009): (1) to minimize the work overload or uncompleted work; and (2) to evenly distribute the material requirements on planning horizon. This variant also includes working conditions for human resources working on the stations in terms of occupancy and activity.

Specifically, it is proposed and evaluated a new mathematical model for the Mixed-Model Sequencing Problem with Work Overload Minimisation (MMSP-W) with serial workstations, with variable activity or work pace of workers, with saturation limitations and with regularity of cumulative production. The model is an extension of the $M 4 \cup 3_{-} \dot{\alpha} I_{-} \eta$ model proposed by Bautista et al. (2015).

\section{The sequencing of mixed-model in JIT systems}

Since the success of Japanese companies after the crisis of the seventies, production systems known as Just In Time (JIT), whose origin is in Toyota, have boomed spectacularly. Indeed, the JIT philosophy, also called Douki Seisan (DS) by Nissan, governs most of actual flexible production systems with MMALs.

This production philosophy translates into a system that produces just what is needed, when is needed, with excellent quality and without wasting the resources of the system. Therefore, one of its general principles is to keep as regular as possible the consumption rates of components in all stations. Principle that also promotes the balance of workloads required to the stations (Pinedo, 2009).

Hence, to satisfy the industrial need of keeping as constant as possible the component consumptions, we use the properties from maintaining the production mix over the manufacturing of products (Bautista et al., 2012). Let $X_{i, t}^{*}$ be the number of units of product type $i \in I$, of a total of $t$ units that should ideally be manufactured to maintain the production mix:

$$
X_{i, t}^{*}=\frac{d_{i}}{T} \cdot t \quad(i=1, \ldots,|I| ; t=1, \ldots, T)
$$

Cumulative production, which must be integer, can be limited between closest values tothe ideal cumulative production that maintains the production mix. 


$$
\left\lfloor\frac{d_{i}}{T} \cdot t\right\rfloor \leq X_{i, t} \leq\left\lceil\frac{d_{i}}{T} \cdot t\right\rceil \quad(i=1, \ldots,|I| ; t=1, \ldots, T)
$$

Specifically, when $t$ units have been sequenced, the cumulative production of product types is limited to the proportion of each type in the production plan. Thereby, the production mix will be kept throughout the sequence as far as possible and both the component consumption and work required will be regular. Thus, the $M M S P-W$ will be in line with another classic problem of literature, such as the Product Rate Variation (PRV) proposed by Miltenburg (1989).

\section{The MMSP-W with Labor Conditions and Production Mix Restrictions}

From the $M 4 \cup 3_{-} \dot{\alpha} I_{-} \eta$, (Bautista et al., 2015), and the $M 4 \cup 3_{-} p m r$ (Alfaro, 2015) models, a new model to improved both productivity and working conditions is proposed. This model incorporates limitations established by collective agreements on the level of occupation or saturation of operators; the chance to complete more required work by increasing the activity factor of operators; and the cumulative production limitation to keep production mix along the sequence. The parameters and variables of $M 4 \cup 3_{-} p m r_{-} \dot{\alpha} I_{-} \eta$ are the following:

Parameters

$K$ Set of workstations, arranged in series, that makes up the line $(k=1, \ldots,|K|)$.

$b_{k} \quad$ Number of homogeneous processors at workstation $k(k=1, \ldots,|K|)$.

I Set of product types that must be manufactured in the line $(i=1, \ldots,|I|)$.

$d_{i} \quad$ Programmed demand of the product type $i(i=1, \ldots,|I|)$.

$p_{i, k}$ Processing time (at normal activity) required by one unit of a product type $i(i=1, \ldots,|I|)$ at workstation $k(k=1, \ldots,|K|)$ for each homogeneous processor.

$T \quad$ Total demand. Obviously: $\sum_{i=1}^{|T|} d_{i}=T$.

$t \quad$ Position index in the sequence $(t=1, \ldots, T)$.

$c \quad$ Cycle time. Normal time assigned to each homogeneous processor in the workstations $(k=1, \ldots,|K|)$ to process any product unit.

$l_{k} \quad$ Temporal window. Maximum time that each homogeneous processor of workstation $k$ $(k=1, \ldots,|K|)$ is allowed to work on any unit of product; once the cycle has been completed, the maximum time that a unit of product can be retained in station $k$ is $l_{k}-c>0$.

$\dot{\alpha}_{k, t}$ Dynamic factor of the work pace or activity associated with the $t^{\text {th }}$ operation of the product sequence $(t=1, \ldots, T)$ at the workstation $k(k=1, \ldots,|K|)$. 
$\dot{\alpha}_{t} \quad$ Dynamic factor of the work pace or activity associated with period $t(t=1, \ldots, T+|K|-1)$ of the extended workday. This extended workday includes $T$ manufacturing cycles (total demand) and $|K|-1$ additional cycles, which are required to complete the required work by the production units in all the workstations. Note that if we associate the same dynamic factor with each moment of the workday in all of the workstations, we will have: $\dot{\alpha}_{k, t}=\dot{\alpha}_{t+k-1}(k=1, \ldots,|K| ; t=1, \ldots, T)$.

$\eta_{m e d}^{\infty}$ Allowable average saturation by the processors of each workstation $(k=1, \ldots,|K|)$.

$\eta_{\max }^{\infty}$ Allowable maximum saturation by the processors of each workstation $(k=1, \ldots,|K|)$.

Variables

$x_{i, t}$ Binary variable equal to 1 if the product unit $i(i=1, \ldots,|I|)$ is assigned to the position $t$ $(t=1, \ldots, T)$ of the sequence and 0 otherwise.

$\hat{s}_{k, t}$ Relative start instant. Positive difference between the start instant and the earliest start instant of the $t^{\text {th }}$ operation at workstation $k(k=1, \ldots,|K|)$. It is fulfilled

$\hat{s}_{k, t}=\left[s_{k, t}-(t+k-2) c\right]^{+}$

$v_{k, t}$ Processing time applied by each homogeneous processor (at normal activity) to the $t^{\text {th }}$ product unit sequenced in the workstation $k(k=1, \ldots,|K|)$.

$\hat{v}_{k, t}$ Processing time reduced by the dynamic factor of activity $\dot{\alpha}_{k, t}$. It is established that $v_{k, t}=\dot{\alpha}_{k, t} \cdot \hat{v}_{k, t}(k=1, \ldots,|K| ; t=1, \ldots, T)$.

$w_{k, t}$ Work overload measured in units of time (at normal activity) generated by the $t^{\text {th }}$ product unit sequenced in each homogeneous processor of the workstation $k(k=1, \ldots,|K|)$.

And the mathematical model, $M 4 \cup 3_{-} p m r_{-} \dot{\alpha} I_{-} \eta$, is the following:

\begin{tabular}{lc}
\hline & $\min W=\sum_{k=1}^{|K|}\left(b_{k} \sum_{t=1}^{T} w_{k, t}\right) \Leftrightarrow \max V=\sum_{k=1}^{|K|}\left(b_{k} \sum_{t=1}^{T} v_{k, t}\right)$ \\
\hline$\sum_{t=1}^{T} x_{i, t}=d_{i}$ & $(i=1, \ldots,|I|)$ \\
$\sum_{i=1}^{|I|} x_{i, t}=1$ & $(t=1, \ldots, T)$ \\
$v_{k, t}+w_{k, t}=\sum_{i=1}^{|I|} p_{i, k} \cdot x_{i, t}$ & $(k=1, \ldots,|K| ; t=1, \ldots, T)$ \\
$\dot{\alpha}_{t+k-1} \cdot \hat{v}_{k, t}-v_{k, t}=0$ & $(k=1, \ldots,|K| ; t=1, \ldots, T)$ \\
$\sum_{t=1}^{T} \hat{v}_{k, t} \leq \eta_{m e d}^{\infty} \cdot c \cdot T$ & $(k=1, \ldots,|K|)$ \\
$\hat{v}_{k, t} \leq \eta_{\max }^{\infty} \cdot c$ & $(k=1, \ldots,|K| ; t=1, \ldots, T)$ \\
$\left.\sum_{\tau=1}^{t} x_{i, \tau} \geq \mid \frac{d_{i}}{T} \cdot t\right]$ & $(i=1, \ldots,|I| ; t=1, \ldots, T)$ \\
$\sum_{\tau=1}^{t} x_{i, \tau} \leq\left|\frac{d_{i}}{T} \cdot t\right|$ & $(i=1, \ldots,|I| ; t=1, \ldots, T)$ \\
$\hat{s}_{k, t} \geq \hat{s}_{k, t-1}+\hat{v}_{k, t-1}-c$ & $(k=1, \ldots,|K| ; t=2, \ldots, T)$ \\
\hline
\end{tabular}




\begin{tabular}{lr}
\hline$\hat{s}_{k, t} \geq \hat{s}_{k-1, t}+\hat{v}_{k-1, t}-c$ & $(k=2, \ldots,|K| ; t=1, \ldots, T)$ \\
$\hat{s}_{k, t}+\hat{v}_{k, t} \leq l_{k}$ & $(k=1, \ldots,|K| ; t=1, \ldots, T)$ \\
$\hat{s}_{k, t}, v_{k, t}, \hat{v}_{k, t}, w_{k, t} \geq 0$ & $(k=1, \ldots,|K| ; t=1, \ldots, T)$ \\
$x_{i, t} \in\{0,1\}$ & $(i=1, \ldots,|| ; \mid t=1, \ldots, T)$ \\
$\hat{s}_{1,1}=0$ &
\end{tabular}

The objective function (1.3) denotes the equivalence between the minimization of the total dynamic work overload $(W)$ and the maximization of the total completed work $(V)$. The sets (1.4) and (1.5) make sure the satisfaction of the demand plan and the assigment of each product to one position of the sequence. The set (1.6) establishes the relationship between the required processing time, $p_{i, k}$, the applied processing time at normal work pace, $v_{k, t}$, and the work overload generated at workstations, $w_{k, t}$, considering the same work pace factor for all workstations. The set (1.7) reduces the processing times, $\hat{v}_{k, t}$, by the work pace factor, $\dot{\alpha}_{t}$. Constraints (1.8) and (1.9) limit the average and maximum saturation values of processors. The sets (1.10) and (1.11) regulate the cumulative productions by product types to preserve the production mix. The sets (1.12)(1.14) determine the relative start instants of the operations at workstations. Constraints (1.15) and (1.16) establish the non-negativity and binarity of the variables and (1.17) fix the start instant of the operations.

\section{Computational Experience}

To assess the impact of production mix restrictions on the MMSP-W when the saturation limitations and activation of processors are considered, a case study linked to the engine assembly line of Nissan in Barcelona is used. Specifically, a production plan corresponding with a total daily demand of 270 engines, which are grouped into 9 types depending on the type of vehicle: $4 \times 4$, vans and trucks. The line has $|K|=21$ workstations, with a cycle time of $c=175 \mathrm{~s}$ and a time window $^{2}$ of $l_{k}=195 \mathrm{~s}$ by station and processor ${ }^{3}$.

Besides, taking into account the collective agreement of Nissan it is possible to set the allowable values for the saturation levels, such as $\eta_{\max }^{\infty}=1.20$ and $\eta_{m e d}^{\infty}=0.95 ;$ and the values for the minimum $\left(\alpha_{i, k}^{\circ}=0.90\right)$, normal $\left(\alpha_{i, k}^{N}=1.00\right)$ and maximum $\left(\alpha_{i, k}^{*}=1.20\right)$ work pace factor allowed by the company.

\footnotetext{
${ }^{2}$ Maximum time that a unit may be retainedfor a workstation.

${ }^{3}$ Team of two workers with identical skills and tools.
} 
The above together with the relationship between the operators' performance and their level of activation has allowed us to define three functions for the dynamic work pace factor (stepped, triangular and trapezoidal) (Bautista et al., 2015). Figure 1 shows the stepped function for the study case in question.

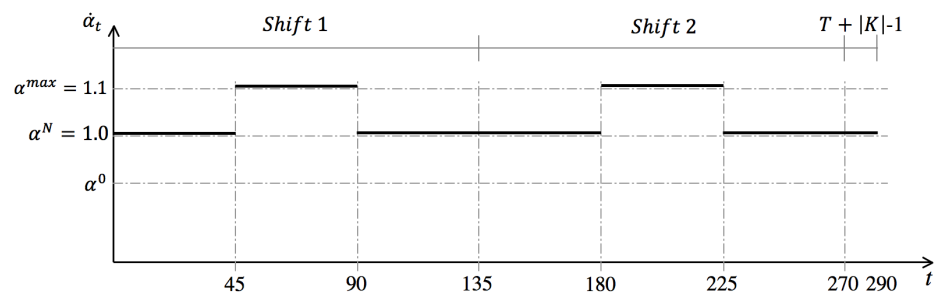

Fig. 1 Stepped function for the work pace factor, $\dot{\alpha}^{S}$, considering the case study from Nissan.

The $M 4 \cup 3_{-} p m r_{-} \dot{\alpha} I_{-} \eta$ model was running in the Gurobi v4.6.1 solver, on a Apple Macintosh iMac computer with an Intel Core i72.93 GHz processor and 8 GB of RAM using MAC OS X 10.6.7., limiting the CPU time to 2 hours.

Once the results have been obtained, these were compared with the results given by: the $M 4 \cup 3$ model that does not consider activation nor saturation, nor regular production; the $M 4 \cup 3_{-} p m r$ model that only considers the regular production; the $M 4 \cup 3 \_\dot{\alpha} I$ model that considers the variable work pace factor; the $M 4 \cup 3 \_\eta$ model with saturation constraints; and the $M 4 \cup 3_{-} \dot{\alpha} I_{-} \eta$ model that is a combination between the above two.Thereby it is possible to evaluate the influence of the labor conditions and the regular production constraints within the $M M S P-W$. To do this, the following indicatorsare defined:

$$
\begin{gathered}
R P D_{1}=\frac{\psi\left(S_{M 4 \cup 3}^{*}\right)-\psi\left(S_{M 4 \cup 3_{-} p m r}^{*}\right)}{\psi\left(S_{M 4 \cup 3}^{*}\right)} \cdot 100 \quad(\psi \in \Psi) \\
R P D_{2}=\frac{\psi\left(S_{M 4 \cup 3}^{*}\right)-\psi\left(S_{M 4 \cup 3_{\_} \dot{\alpha} I(f)}^{*}\right)}{\psi\left(S_{M 4 \cup 3}^{*}\right)} \cdot 100 \quad(\psi \in \Psi ; f \in \mathfrak{I}) \\
R P D_{3}=\frac{\psi\left(S_{M 4 \cup 3}^{*}\right)-\psi\left(S_{M 4 \cup 3 \_}^{*}\right)}{\psi\left(S_{M 4 \cup 3}^{*}\right)} \cdot 100 \quad(\psi \in \Psi) \\
R P D_{4}=\frac{\psi\left(S_{M 4 \cup 3}^{*}\right)-\psi\left(S_{M 4 \cup 3 \_\dot{\alpha} I(f) \_\eta}^{*}\right)}{\psi\left(S_{M 4 \cup 3}^{*}\right)} \cdot 100 \quad(\psi \in \Psi ; f \in \mathfrak{I}) \\
R P D_{5}=\frac{\psi\left(S_{M 4 \cup 3}^{*}\right)-\psi\left(S_{M 4 \cup 3_{-} p m r_{-} \dot{\alpha} I(f) \_\eta}^{*}\right)}{\psi\left(S_{M 4 \cup 3}^{*}\right)} \cdot 100 \quad(\psi \in \Psi ; f \in \mathfrak{I}) \\
R P D_{6}=\frac{\psi\left(S_{M 4 \cup 3 \_\dot{\alpha} I(f) \_\eta}^{*}\right)-\psi\left(S_{M 4 \cup 3_{-} p m r_{-} \dot{\alpha} I(f) \_\eta}^{*}\right)}{\psi\left(S_{M 4 \cup 3_{-} \dot{\alpha} I(f) \_\eta}^{*}\right)} \cdot 100 \quad(\psi \in \Psi ; f \in \mathfrak{I})
\end{gathered}
$$


Where $\Psi=\left\{W, \Delta_{Q}(X), \Delta_{Q}(P)\right\}$ is the set of functions evaluated for each solution obtained by a specific model, $S_{M}^{*}$; and $\mathfrak{I}=\left\{\dot{\alpha}^{S}, \dot{\alpha}^{T}, \dot{\alpha}^{Z}\right\}$ is the set of functions for the dynamic work pace factor.

Table 1 Comparison between the work overload values, $W(s)$, obtained with the proposed model, $M 4 \cup 3_{-} p m r_{-} \dot{\alpha} I_{-} \eta$, and those obtained with the reference models from literature.

\begin{tabular}{|c|c|c|c|c|c|c|c|c|}
\hline \multirow{2}{*}{$M 4 \cup 3$} & \multirow{2}{*}{$M 4 \cup 3_{-} p m r$} & \multirow{2}{*}{$\begin{array}{l}M 4 \cup 3 \_\dot{\alpha} I \\
\dot{\alpha}^{S}, \dot{\alpha}^{T}, \dot{\alpha}^{Z}\end{array}$} & \multirow{2}{*}{$M 4 \cup 3 \_\eta$} & \multicolumn{2}{|c|}{$M 4 \cup 3 \_\dot{\alpha} I \_\eta$} & \multicolumn{3}{|c|}{$M 4 \cup 3_{-} p m r_{-} \dot{\alpha} I_{-} \eta$} \\
\hline & & & & $\dot{\alpha}^{S}$ & $\dot{\alpha}^{T} \dot{\alpha}^{Z}$ & $\dot{\alpha}^{S}$ & $\dot{\alpha}^{T}$ & $\dot{\alpha}^{Z}$ \\
\hline 187 & 186 & 0 & 12315 & 4602 & 7960 & 4669 & 839 & 0 \\
\hline
\end{tabular}

Table 2 Relative percentage deviations (\%) of work overload $(W)$ and non-regularity of both cumulative production $\left(\Delta_{Q}(X)\right)$ and required work $\left(\Delta_{Q}(P)\right)$.

\begin{tabular}{|c|c|c|c|c|c|c|c|c|c|c|c|c|}
\hline \multirow{2}{*}{$\psi \in \Psi$} & \multirow{2}{*}{$R P D_{1}$} & \multicolumn{3}{|c|}{$R P D_{2}$} & \multirow{2}{*}{$P D_{3}$} & \multicolumn{3}{|c|}{$R P D_{4}$} & \multicolumn{2}{|l|}{$R P D_{5}$} & \multicolumn{2}{|l|}{$R P D_{6}$} \\
\hline & & $\dot{\alpha}^{S}$ & $\dot{\alpha}^{T}$ & $\dot{\alpha}^{Z}$ & & $\dot{\alpha}^{S}$ & $\dot{\alpha}^{T}$ & $\omega$ & $\dot{\alpha}^{S} \quad \dot{\alpha}^{T}$ & $\dot{\alpha}^{Z}$ & $\begin{array}{ll}\dot{\alpha}^{S} & \dot{\alpha}^{T} \quad o\end{array}$ & $\dot{\alpha}^{Z}$ \\
\hline$w$ & 0.5 & 100 & 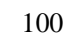 & 100 & & -23 & -325.7 & 100 & $-2396.7-348.7$ & 100 & $\begin{array}{lll}-1.5 & -5.4 & 0\end{array}$ & 0.0 \\
\hline$(X)$ & 97.7 & 14.5 & 31.2 & 43.4 & 27.4 & -83.5 & -213.8 & -36.7 & $7.797 .7 \mathrm{~s}$ & 97.7 & 98.899 .39 & 98.3 \\
\hline$W$ & 96.4 & -61.8 & -75.4 & -56.8 & -19.5 & -193.3 & $-467.5-$ & -224.9 & S95.495.7 & 96.3 & 98.499 .29 & 98.9 \\
\hline
\end{tabular}

Tables 1 and 2 show the results. First, Table 1, where the overall work overloads values are collected, clearly shows the negative effect of saturation limitations on the work overload. Indeed, when the saturation constraints are added into the model the work overload considerably increase $(187 \mathrm{~s}$ versus $12315 s$ ). However, when the activity factor is increased by $3.33 \%$ (stepped function), 5\% (triangular function) and $6.66 \%$ (trapezoidal function), on average, the work overload is reduced. The incorporation of regularity does not produce an important increase of work overload.

On the other hand, Table 2 shows the relative percentage deviations in regard with the work overload, the cumulative production discrepancy (1.24) and the required work discrepancy (1.25).

$$
\begin{gathered}
\Delta_{Q}(X)=\sum_{t=1}^{T} \sum_{i=1}^{|I|}\left(X_{i, t}-X_{i, t}^{*}\right)^{2}=\sum_{t=1}^{T} \sum_{i=1}^{|I|}\left[\left(\sum_{\tau=1}^{t} x_{i, \tau}\right)-\left(t \cdot \frac{d_{i}}{T}\right)\right]^{2} \\
\Delta_{Q}(P)=\sum_{t=1}^{T} \sum_{k=1}^{|K|}\left(P_{k, t}-P_{k, t}^{*}\right)^{2}=\sum_{t=1}^{T} \sum_{k=1}^{|K|}\left[\left(b_{k} \sum_{i=1}^{|I|} p_{i, k}\left(\sum_{\tau=1}^{t} x_{i, \tau}\right)\right)-\left(t \cdot \frac{b_{k}}{T} \sum_{i=1}^{|I|} p_{i, k} \cdot d_{i}\right)\right]^{2}
\end{gathered}
$$

The incorporation of restrictions to regulate the production mix improved the regularityof sequences in terms of cumulative production and work required to the line by more than $95 \%$,. This gain in regularity offsets the overload increase of 
$M 4 \cup 3_{-} p m r_{-} \dot{\alpha} I_{-} \eta$ model in regard with $M 4 \cup 3_{-} \dot{\alpha} I_{-} \eta$ model, with values of $1.5 \%$ and $5.4 \%$, when the stepped or triangular function are considered.

\section{Conclusions}

Simultaneous integration of JIT concepts and human resources aspects has led to $M 4 \cup 3_{-} p m r_{-} \dot{\alpha} I_{-} \eta$ model. This model, in addition to complying with legal provisions, contained in the collective agreements, on the level of occupancy of the workers, can eliminate the work overload by increasing the factor of normal activity of the workers at certain moments of their working day. This makes possible to respect working conditions without compromising the line productivity. Moreover, the properties of maintaining constant the production mix favour the decrease of non-regularity of cumulative production and required work and therefore the regular comsumption of components is favoured.

Specifically, with an average increase of $6.66 \%$ of normal activity, the overall work overload is completely eliminated while the saturation conditions are satisfayed. In addition, the regularity of cumulative production and required work improve by $98.3 \%$ and $98.9 \%$ respectively the solution given by the reference model $M 4 \cup 3_{-} \dot{\alpha} I_{-} \eta$.

In future research the gains will be economically evaluated in order to verify if the gains in regularity are compensated with the loss in completed work when the stepped, $\dot{\alpha}^{S}$, or triangular, $\dot{\alpha}^{T}$, functions are considered.

\section{References}

Alfaro R (2015) Modelado y resolución de variantes del problema de secuenciación de modelos mixtos con minimización de la sobrecarga (MMSP-W) con factores de actividad y regularidad en la producción. $\mathrm{PhD}$ thesis. Universitat Politècnica de Catalunya. DOI: $10.13140 / 2.1 .1691 .9362$

Bautista J, Alfaro R, Batalla C et al (2015) Mixed-model sequencing problem improving labour conditions. In: Cortés, P. et al. (Eds.), Enhancing Synergies in a Collaborative Environment. Series: Lecture Notes in Management and Industrial Engineering. Springer, ISBN: 978-3319-14077-3, Chapter 1:3-10.

Bautista J, Cano A, Alfaro R (2012) Modeling and solving a variant of the mixed-model sequencing problem with work overload minimisation and regularity constraints. An application in Nissan's Barcelona Plant. Expert Syst. Appl. 39(12):11001-11010.

Boysen N, Fliedner M, Scholl A (2009) Sequencing mixed-model assembly lines: Survey, classification and model critique. Eur. J. Prod. Econ. 192(2):349-373.

Miltenburg J (1989) Scheduling mixed-model assembly lines for just-in-time production systems. Manage. Sci. 35(2):192-207.

Pinedo ML (2009) Planning and Scheduling in Manufacturing and Services. Springer Series in Operations Research and Financial Engineering. 2nd ed, XVIII, 536. ISBN: 978-1-44190909-1 